\title{
EL PROJECT FINANCE, LAS ASOCIACIONES PÚBLICO-PRIVADAS Y LA BRECHA DE INFRAESTRUCTURA en el Perú
}

\author{
Pierre Nalvarte Salvatierra* \\ Universidad de Lima, Lima, Perú \\ Recibido: 18/11/2020 Aprobado: 25/11/2020 \\ doi: https://doi.org/10.26439/iusetpraxis2021.n053.4971
}

\begin{abstract}
RESUMEN. La necesidad de cerrar la brecha de infraestructura en el Perú es, creemos, indiscutible. Y uno de los mecanismos más apropiados para el desarrollo de la infraestructura y la prestación de servicios públicos de calidad, sostenibles en el tiempo, son las asociaciones público-privadas (APP). En ese sentido, uno de los aspectos más destacados de las APP es el tipo de financiamiento especial que se utiliza en tales proyectos, conocido como project finance. En el presente trabajo, se analizan brevemente las principales características del project finance, la brecha de infraestructura y la manera concreta como, mediante las APP, se ha impulsado la ejecución de obras de infraestructura pública, utilizando el project finance como modalidad de financiamiento.
\end{abstract}

PALABRAS CLAVE: project finance / asociaciones público-privadas / brecha de infraestructura / Perú

\section{PROJECT FINANCE, PUBLIC-PRIVATE PARTNERSHIPS AND THE INFRASTRUCTURE GAP IN PERU}

\begin{abstract}
The need to close the infrastructure gap in Peru is, we believe, indisputable. Moreover, one of the most appropriate mechanisms for developing infrastructure and providing quality public services, sustainable over time, are PPPs. In this sense, one of the most outstanding aspects of PPPs is the type of special financing used in this type of project, known as Project Finance. In this paper, we will briefly analyze the main characteristics of Project Finance, the infrastructure gap, and the concrete way through PPPs, public infrastructure works has been promoted, using Project Finance as a financing modality.
\end{abstract}

KEYWORDS: project finance / public-private partnerships / infrastructure gap / Peru

\footnotetext{
*Abogado por la Universidad de Lima, Perú. MBA por ESAN. Máster en Derecho de la Contratación Pública de la Universidad Castilla-La Mancha, España. Profesor de la Facultad de Derecho de la Universidad de Lima.
} 


\section{INTRODUCCIÓN}

Consideramos que todos los que enseñamos y escribimos debemos hacer un esfuerzo por explicar las cosas con la mayor claridad posible. En cierta manera, quien domina una materia puede explicarla con sencillez o, dicho en otras palabras, quien no es capaz de explicar con claridad un concepto es porque, quizá, no lo ha entendido completamente.

Hoy en día, el estilo grandilocuente persigue solo el lucimiento de quien ejerce el arte de hablar con elocuencia; y lejos de constituir un instrumento de orientación e intercambio comunicativo bilateral, reduce la capacidad de presentarse con éxito ante los oyentes, puesto que en la relación público-orador resulta vital la dicotomía comprender-ser comprendido, pero sin olvidar el precepto de que se impone hacerles pensar, no exigirles entender. (Jiménez, 2015, p. 274)

Lo contrario de explicar algo con claridad, tanto al enseñar como al escribir, es, por ejemplo, repetir lo que dice un autor o repetir lo que dicen varios autores y pretender que los alumnos repitan y memoricen sin comprender a cabalidad el concepto o la institución jurídica de la cual se trata.

En el presente artículo, es nuestro propósito definir con la mayor sencillez lo que significa el project finance y luego de ello, si la extensión del trabajo nos lo permite, explicar cómo este mecanismo ha sido utilizado en el Perú para la ejecución de obras de infraestructura pública y servicios públicos.

\section{EL CORPORATE FINANCE}

En primer lugar, para entender a cabalidad el concepto de project finance, debemos retroceder un paso y hablar del corporate finance o financiamiento corporativo.

Una empresa requiere financiamiento corporativo, por ejemplo, deuda de largo plazo, por diversos motivos: implementar una nueva sucursal, adquirir maquinaria, overhauling de la maquinaria, etcétera. Lo que hace esta empresa, típicamente, es solicitar un préstamo a un banco. Lo que hace el banco, comúnmente, es analizar los estados financieros de la empresa. Los estados financieros más importantes son el estado de resultados (antes conocido como estado de ganancias y pérdidas) y el estado de situación patrimonial (antes conocido como balance general).

El estado de resultados es relevante para conocer la capacidad de generación de flujo de caja de la empresa. El estado de situación patrimonial sirve para conocer, de un lado, cuáles son los activos de la empresa, los cuales pueden servir, eventualmente, como una garantía del préstamo (por ejemplo, el terreno en donde están ubicadas las oficinas o la fábrica de la empresa, a veces, puede constituir una garantía excelente, dependiendo de la ubicación); y, de otro lado, permite saber cuál es el nivel de endeudamiento de la empresa. Si la empresa tiene poca deuda, se considera menor riesgo. Si 
la empresa ya tiene un nivel de endeudamiento significativo (por ejemplo, $80 \%$ deuda y $20 \%$ patrimonio), se percibe un riesgo alto y ello podría conducir a dos situaciones: otorgamiento del préstamo con un interés más alto o no otorgamiento del préstamo.

Llamemos a la empresa ABC. Supongamos que el estado de situación patrimonial de la empresa era el que se muestra en la figura 1.

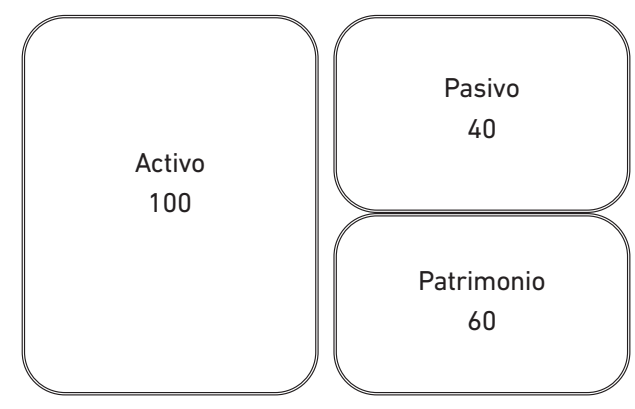

Figura 1. Estado de situación patrimonial de la empresa ABC (expresado en millones) Elaboración propia

Como se puede apreciar, en el lado del pasivo y patrimonio, el pasivo representa el $40 \%$ y el patrimonio el $60 \%$. Convertido en ratio, se hablaría de un ratio deuda/capital de 0,67 (resultado de dividir 40/60). Se podría decir que es una empresa con bajo nivel de endeudamiento.

Luego, la empresa $A B C$ pide un préstamo de 30 millones para la compra de nueva maquinaria (ampliación de la capacidad de producción, quizá una nueva línea de negocio). El banco le otorga el préstamo. El nuevo estado de situación patrimonial será el que se muestra en la figura 2.

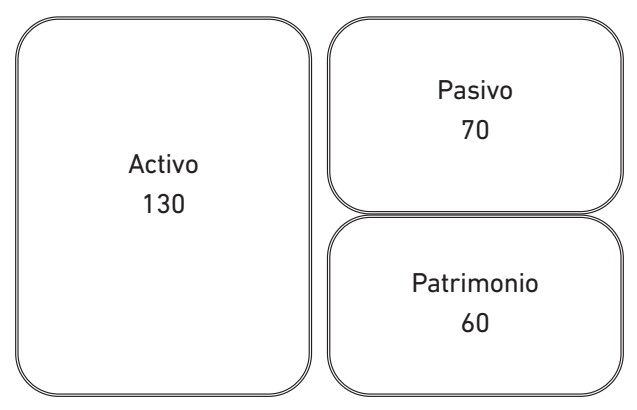

Figura 2. Estado de situación patrimonial de la empresa ABC (expresado en millones) después de recibir un préstamo

Elaboración propia 
Como se puede apreciar, ha cambiado el nivel de endeudamiento de la empresa. Su ratio deuda/capital es ahora de 1,17 (resultado de dividir 70/60). Un ratio deuda/capital mayor a 1 indica que la deuda es mayor que el patrimonio. Esto no es malo necesariamente. Es común que las empresas tengan un ratio deuda/capital mayor a 1. En general, el problema ocurre cuando el ratio deuda/capital es mayor a 2,5 o 3,0.

Lo que hemos querido resaltar en este ejemplo es que con el corporate finance se afecta el balance de una empresa, así como su ratio deuda/capital y su capacidad para adquirir, más adelante, nuevos endeudamientos. Todo ello sin mencionar que la empresa podría haber puesto activos en garantía y que su accionar podría estar restringido por los términos del contrato de financiamiento (restricciones a la distribución de dividendos, por ejemplo).

\section{EL PROJECT FINANCE}

Ahora bien, el project finance consiste en una forma totalmente distinta de financiamiento. Se aplica cuando se quiere realizar un proyecto independiente. Supongamos que la empresa $A B C$ es una empresa constructora. Tiene experiencia construyendo carreteras, hospitales y edificios; y ahora se le presenta una nueva oportunidad de negocio: la construcción de una carretera en el norte del Perú. La inversión estimada es de 30 millones. Pero este proyecto tiene una particularidad: se trata de una concesión. Es decir que el Estado no le pagará la obra con recursos del tesoro público, sino que más bien la empresa deberá recuperar sus costos de inversión (CAPEX o capital expenditures) con el cobro de un peaje. Obviamente, sus gastos de operación y mantenimiento (OPEX u operational expenditures) también los deberá cubrir con el mismo peaje.

Entonces, la empresa $A B C$, en lugar de solicitar un financiamiento tradicional para la ejecución de dicha obra, decide aplicar la figura del project finance. Esto es, constituye una nueva empresa, a la cual llamaremos Concesionaria $A B C$, con el único propósito de tomar a su cargo la concesión del proyecto. Es decir, se trata de una sociedad de propósito exclusivo (SPE), también conocida como SPV (special purpose vehicle) por ser el "vehículo" a través del cual la empresa ABC realizará la inversión.

Luego, la SPV denominada Concesionaria ABC será la que solicite el financiamiento para la ejecución de la obra. Pero, y he aquí la característica especial del project finance, ¿cómo será posible que una entidad financiera le otorgue el préstamo a una empresa nueva que no tiene historial y tampoco tiene activos con los cuales garantizar el pago del préstamo?

Recordemos que, en el ejemplo anterior, lo que el banco analiza para otorgar el préstamo a la empresa $A B C$, entre otras cosas, es su estado de resultados para evaluar cómo ha evolucionado el flujo de caja de la empresa, así como el estado de situación patrimonial, 
para identificar tanto los activos como el grado de endeudamiento. Sin embargo, en el caso de la Concesionaria $A B C$, de nada sirve analizar el estado de resultados, puesto que se trata de una empresa nueva. Y esta tampoco cuenta con grandes activos "físicos" que puedan servir para garantizar el pago de un préstamo, como terrenos, edificios o maquinarias.

¿Cómo es posible, entonces, que una entidad financiera le otorgue el préstamo? Lo que sucede en el project finance es que se evalúa el futuro, y no el pasado. Se evalúa un proyecto específico, y no todas las actividades de una empresa. Así, por ejemplo, en este caso, se evaluaría el contrato de concesión, el tráfico de la carretera (tráfico histórico si fuera una carretera existente o tráfico proyectado si fuera una carretera nueva), así como los factores de riesgo de la concesión, qué ocurriría ante un caso fortuito o de fuerza mayor, qué sucedería en caso de cambios en las normas legales que afecten la concesión, cómo mitigar el riesgo de diseño, de construcción, de calidad del servicio, etcétera.

En definitiva, se evalúa el proyecto en sí: el flujo de caja proyectado de la concesión, sus riesgos, las garantías que, de ser el caso, otorgue el Estado (por ejemplo, una garantía de ingreso mínimo), los derechos de los financistas (como el derecho de cambiar al concesionario) y, con base en todo ello, se puede otorgar el financiamiento.

Las experiencias investigadas por Yescombe (2013) permiten concluir que el project finance es el método más adecuado de financiamiento a largo plazo para proyectos de capital intensivo, donde la inversión apalancada tiene un flujo de caja relativamente predecible. Yescombe (2013) concluye, además, que el continuo mejoramiento del project finance y de su estructuración han desempeñado un papel importante en la modernización de los mecanismos necesarios para la provisión de los fondos requeridos en el desarrollo de proyectos de inversión a largo plazo. (Reátegui et al., 2018, p. 17)

En el project finance, es común que el nivel de endeudamiento de la SPV sea relativamente alto. Así, por ejemplo, un concesionario SPV puede tener un endeudamiento de 80 a $90 \%$ y el patrimonio solamente representa el 20 o el $10 \%$ de los fondos. Esto es algo "normal" en el project finance, dado que los prestamistas están tomando todas las precauciones posibles para asegurar el retorno de su préstamo.

Supongamos, en el caso propuesto, que se obtendrá un financiamiento por el $90 \%$ del monto de inversión, esto es, un financiamiento por 27 millones. En consecuencia, la empresa ABC (la matriz) tendría que colocar un capital de 3 millones. Con ello, el estado de situación patrimonial de la SPV quedaría conformado como se observa en la figura 3. 


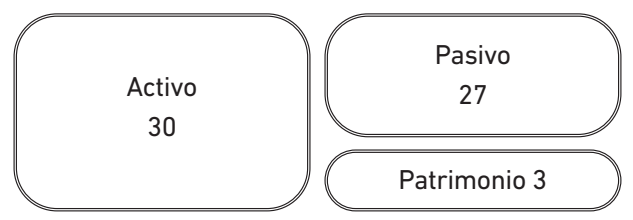

Figura 3. Estado de situación patrimonial de la Concesionaria ABC (expresado en millones) después de obtener el financiamiento

Elaboración propia

Una de las grandes ventajas del project finance y a la vez una de sus características principales es que a través de este mecanismo se consigue un financiamiento sin afectar o afectando mínimamente el balance de la empresa matriz. En efecto, el financiamiento otorgado a la SPV (Concesionaria $\mathrm{ABC}$ ) no se registra en el estado de situación patrimonial de la empresa ABC. Por ello, se habla de un financiamiento "fuera de balance". Al no considerarse el financiamiento en el balance de la matriz, no afecta su ratio deuda/ capital, con lo cual no afecta la capacidad de la matriz de obtener nuevos financiamientos.

Permite financiarse fuera de balance, en unas condiciones adecuadas de importe, plazo y precio. Las agencias de rating suelen mantener la valoración crediticia de los sponsors al no considerar como deuda de la matriz la asumida por las sociedades vehículo. (Casanovas, 2016, p. 90)

Esto es, en pocas palabras, el project finance. Una modalidad de financiamiento en la que una empresa o un grupo de empresas que se unen en consorcio pueden realizar un proyecto, generalmente un proyecto de infraestructura (pública o privada), a través de una vehículo especialmente diseñado para ello (la SPV), sin afectar el balance de la o las empresas que impulsan el proyecto (denominados sponsors), sobre la base de la garantía de uno o varios contratos (si es un proyecto privado, puede ser un contrato take or pay; si es un proyecto público, típicamente un contrato de concesión), con la gran ventaja adicional de que se aísla el riesgo del proyecto (en el peor de los casos, si el proyecto fracasa, la pérdida de los sponsors queda limitada al capital que hubieren aportado).

A nivel internacional, este sistema sirve para financiar una notable gama de inversiones, que abarca desde grandes proyectos de infraestructuras, como la construcción de un túnel o un puerto marítimo, hasta la explotación de recursos naturales, como campos eólicos, de gas o petróleo. Sin duda alguna, el project finance se está consolidando a escala mundial como uno de los métodos de financiación más relevantes y de mayor implantación. (Casanovas, 2016, p. 10)

Ahora bien, habiéndose comprendido el concepto de project finance, cabe señalar que esta modalidad de financiamiento se utiliza para la ejecución de grandes proyectos de infraestructura, tanto públicos como privados. En el presente caso, nos interesan los proyectos de infraestructura pública. 


\section{EL PROJECT FINANCE Y LAS APP EN EL PERÚ}

El marco legal que dio impulso a las concesiones se remonta a la década de 1990. Ya nos hemos extendido en otro trabajo sobre la evolución del marco legal de las concesiones y las APP (Nalvarte Salvatierra, 2018). Hoy en día, la norma principal bajo la cual se ejecutan proyectos de infraestructura pública, en donde se puede aplicar la modalidad de project finance, es el Decreto Legislativo 1362 y su reglamento.

El Decreto Legislativo 1362, dado el 21 de julio del 2018, es la ley a través de la cual se regula el desarrollo de las asociaciones público-privadas (APP o PPP, por sus siglas en inglés) en el Perú a través de diversas modalidades contractuales. La modalidad contractual más utilizada es la concesión.

Una APP es un contrato de largo plazo en el que el sector público acude al sector privado, generalmente, para la construcción, ampliación o mejoramiento de una obra pública de infraestructura, así como para la operación y mantenimiento posterior de esta, delegando en el privado la prestación del servicio público asociado o de un servicio vinculado a un servicio público. La diferencia con el mecanismo de contratación tradicional (Ley de Contrataciones del Estado) es notoria, puesto que en una contratación tradicional el contrato es de corto plazo, solo para un objetivo concreto (el diseño, o la construcción, o el mantenimiento o la prestación de un servicio específico), mientras que en una APP en un solo contrato se engloba tanto el diseño como la construcción, la operación y el mantenimiento dentro de un contrato de largo plazo.

Desde un punto de vista financiero, la diferencia entre la ejecución de una obra bajo el mecanismo tradicional y bajo el esquema de APP es muy grande. En una obra pública típica, el pago se efectúa contra valorizaciones mensuales, sin dejar de mencionar que al principio del contrato se hace un adelanto para el inicio de la obra. Es decir, la necesidad de financiamiento por parte de la empresa constructora es nula o mínima, puesto que recibe un adelanto para el inicio de las obras y tiene pagos mensuales durante la ejecución de estas.

Por el contrario, en el caso de una APP, por su propia naturaleza, la recuperación de la inversión en la ejecución de la obra se realizará en el largo plazo (aunque existen contadas excepciones). Así, por ejemplo, en la concesión del Tramo 5 de la carretera Interoceánica, el pago se hace a través del PAO (pago anual por obras) durante un plazo de 20 años.

While there is no single, internationally accepted, definition of PPP, the essentials of a PPP arrangement which are encapsulated in a PPP Contract are:

- A long-term contract between a private party (the Private Partner) and a government entity (the Contracting Authority);

- For providing a new or existing public asset or service; 
- Under which the Private Partner bears significant risk and management responsibility; and

- Where payments received by the Private Partner are linked to performance.

(The World Bank, 2019, p. 6)

Las APP pueden ser autofinanciadas o cofinanciadas. En el primer caso, se trata de proyectos sostenibles financieramente con los ingresos que generan por sí mismos o que obtienen indirectamente de las tarifas que pagan los usuarios del servicio. El caso típico de una APP autofinanciada es la concesión de una carretera que se sostiene únicamente con lo que se recauda a través del peaje o la concesión de un aeropuerto, como el Jorge Chávez, que se sostiene solo por los ingresos que se obtienen mediante la explotación del aeropuerto (lo que comprende ingresos regulados y no regulados).

Las APP cofinanciadas son aquellas que no pueden sostenerse económicamente con los ingresos que generan o que no generan ingresos propios en absoluto y son pagadas directamente por el Estado. Un ejemplo de APP cofinanciada es la concesión del segundo grupo de aeropuertos de provincia (aeropuertos de Arequipa, Tacna, Juliaca, Ayacucho y Puerto Maldonado), pues esta sí genera ingresos propios, pero requiere que el Estado complemente dichos ingresos para poder recuperar las inversiones ejecutadas. Otro ejemplo de APP cofinanciada es la concesión del sistema de tratamiento de las aguas residuales de la cuenca del lago Titicaca. En este caso, podríamos decir que se trata de una concesión prácticamente $100 \%$ cofinanciada por el Estado, puesto que no genera ingresos propios y los costos de operación y mantenimiento, así como los costos de inversión, son cubiertos con recursos del tesoro público.

\section{FINANCIAMIENTO DE PROYECTOS DE INFRAESTRUCTURA EN EL PERÚ}

Recientemente, se hizo pública la noticia del financiamiento otorgado a Aunor (Autopista del Norte S. A.), concesionario de la Red Vial 4, para la ejecución de las obras comprendidas en su contrato de concesión. El monto de financiamiento asciende a 350 millones de dólares y fue otorgado a través de un crédito sindicado. Esto es, un crédito que no hace una sola entidad financiera, sino un conjunto de entidades financieras quienes, de esta manera, comparten el riesgo. Cabe agregar que la SPV fue constituida por OHL Concesiones S. L., y que los estructuradores del financiamiento fueron Credicorp Capital y Sumitomo Mitsui Banking.

Así como en este ejemplo, otras concesiones viales autosostenibles similares se han otorgado en nuestro país, tales como estas:

- Concesión de la Red Vial 5: Ancón-Huacho-Pativilca (2003)

- Concesión de la Red Vial 6: Puente Pucusana-Cerro Azul-Ica (2005) 
- Concesión de la Autopista del Sol: Trujillo-Chiclayo-Piura-Sullana (2009)

- Concesión del Tramo 2 de la IIRSA Centro (Carretera Central) (2010)

- Concesión de la Panamericana Sur: Ica-frontera con Chile (2013)

En todos estos proyectos, los contratos de concesión han reunido una serie de características principales que hacen a los proyectos susceptibles de recibir financiamiento. Esto es, hacen a los proyectos "bancables". Entre dichas características podemos mencionar:

- Se otorga un derecho de concesión a la SPV que le da derecho a cobrar un peaje a una tarifa determinada durante un plazo claramente establecido en el contrato, suficiente como para recuperar las inversiones.

- Adicionalmente a ello, se otorga una garantía de "ingreso mínimo", en virtud de la cual, durante determinado lapso de tiempo, el Estado asegura al concesionario un ingreso mínimo anual. En caso de que la recaudación caiga por debajo de dicho umbral, el concedente pondrá la diferencia para que el concesionario perciba el ingreso mínimo anual.

- El marco legal permite a los concesionarios (SPV) y a sus accionistas (sponsors) suscribir contratos de garantía con el Estado, en virtud de los cuales el Estado peruano garantiza el cumplimiento de las obligaciones previstas en el contrato.

- Asimismo, el marco legal permite a los concesionarios y a sus accionistas celebrar un convenio de estabilidad jurídica, de tal modo que se "congele" la tasa del impuesto a la renta, la libre convertibilidad de moneda extranjera y la libertad de envío de remesas al exterior, entre otros aspectos.

- En todos los contratos se prevé, de alguna manera, algún tipo de "protección" para los financistas en caso de terminación anticipada del contrato. Esto es, si se resuelve el contrato y el financiamiento todavía no se ha cancelado, el Estado podría asumir el pago del saldo de la deuda, dado que se trata de una infraestructura pública.

- Además, en los contratos se establecen derechos a favor de los financistas que hayan sido previamente calificados como "acreedores permitidos". Estos derechos pueden comprender la hipoteca del derecho de concesión, la entrega de acciones y flujos en garantía, así como el derecho de intervenir en el contrato cumpliendo las obligaciones que no hubiere cubierto el concesionario, cuando ello sea necesario o conveniente para evitar la caducidad de la concesión.

- En todos estos contratos también se ha incluido una cláusula de restablecimiento del equilibrio económico de la concesión. Dicha cláusula se puede invocar cuando debido a un cambio en las normas legales se afecta económicamente a 
la concesión, bien sea incrementado los costos, reduciendo los ingresos o una combinación de ambos.

- No menos importante es que para la obtención del financiamiento en todos estos contratos (y prácticamente en todas las APP, en general) se constituye un fideicomiso de administración de flujos, en donde se deposita la recaudación del peaje. De este modo, se garantiza a los financistas que el dinero recaudado será administrado por un tercero neutral y debidamente autorizado, que velará por que los fondos sean derivados, en primer lugar, a favor de los financistas para el pago del financiamiento y luego al concesionario para cubrir sus costos de operación y mantenimiento.

- Sin perjuicio de todo lo anterior, el contrato contiene diversas disposiciones que garantizan el cumplimiento de las obligaciones por parte del concesionario, la permanencia del socio estratégico, la mitigación de riesgos de caso fortuito y fuerza mayor, por ejemplo, mediante la contratación de seguros, y otros elementos usuales en este tipo de contratos y que son evaluados minuciosamente por los financistas antes de aprobar el financiamiento respectivo.

Sobre la necesidad e importancia de incluir una cláusula de restablecimiento del equilibrio económico financiero en los contratos de APP, consideramos apropiado lo señalado por el Banco Mundial:

An unexpected change in law may make the Private Partner's performance of its contractual obligations easier or less costly, or may make it wholly or partially impossible, delayed or more expensive. Through no fault of its own it may find itself in breach of contract, as well as being unable to earn its expected income and also required to incur additional costs to comply with the change. Some changes in law could, for example, entail significant expenditure if additional capital works are required (e.g. to meet new safety or environmental standards - including legislation in relation to climate change - or to provide mandatory disabled access) and may also reduce full performance of the service while implemented. Similarly, additional taxes may be levied.

Without specific contractual provisions, unless relief is available through noncontractual legal doctrines under the relevant governing law, change in law risk would lie entirely with the Private Partner because it has contracted to provide a specified service at a specified price, and the scope of its obligations and its pricing can only be changed in accordance with the PPP Contract. (The World Bank, 2019, p. 59)

\section{LA BRECHA DE INFRAESTRUCTURA Y LAS APP}

Nuestro país tiene una enorme brecha de infraestructura y de servicios públicos. La brecha de infraestructura de corto plazo supera los 35000 millones de dólares y la de largo plazo, los 110000 millones de dólares (Bonifaz et al., 2020, p. 59). La brecha de 
infraestructura no es un concepto etéreo, es un problema real que nos afecta en la vida diaria. Por ejemplo, la falta de un sistema de transporte masivo nos hace perder miles de horas hombre en Lima y Callao, así como también tiene otros efectos negativos como incrementar la contaminación ambiental (un metro que funciona con electricidad contamina mucho menos que miles de buses o vehículos que funcionan con combustibles fósiles), reducir el valor de las propiedades, aumentar el estrés, la inseguridad, etcétera.

Y este es solo uno de los ejemplos. Otro de los sectores en donde existe una enorme brecha de infraestructura es en el de saneamiento. Por ejemplo, muchas regiones no cuentan con una adecuada planta de tratamiento de aguas residuales (PTAR). Ello significa que se contaminan los ríos, los lagos (como el Titicaca) o el mar, pues se vierten aguas contaminadas que afectan a la población, la flora y la fauna. Y así podríamos seguir extendiendo ejemplos claros y directos de la brecha de infraestructura que afecta nuestras vidas diarias y la competitividad del país, lo que se nota con mayor énfasis hoy en día que vivimos en un mundo comercialmente globalizado.

Por todo ello, creemos que está fuera de discusión que se necesita tomar acciones decididas y eficaces para reducir la brecha de infraestructura. Estas decisiones y políticas de Estado deben ser sostenidas en el tiempo, pues sus beneficios no se verán en el corto plazo, pero son necesarias. En ese sentido, por ejemplo, el Ministerio de Economía y Finanzas publicó en el 2018 el Plan Nacional de Infraestructura para la Competitividad (PNIC) como una herramienta para orientar el accionar del Estado en el largo plazo.

Ahora bien, el cómo abordar la reducción de la brecha de infraestructura puede tomar varias alternativas. Parte de las obras de infraestructura se pueden realizar mediante el modelo tradicional de la contratación pública. Otra parte se puede desarrollar bajo el mecanismo peruano de las obras por impuestos, modalidad que está siendo observada con interés por otros países.

Globally, governments are accountable for the development of infrastructure (both economic and social) and the delivery of basic services in an affordable and inclusive manner. This is irrespective of whether such infrastructure is financed, created and managed by public authorities, or with the involvement of the private sector through public-private partnerships (PPPs).

Nurturing a conducive enabling environment for infrastructure investment is largely the responsibility of governments (both national and sub-national). It is often a key differentiator between countries that successfully scale up infrastructure and those that face challenges in doing so. (Global Infrastructure Hub, 2018, p. 15)

Pero una parte fundamental de las obras de infraestructura y de servicios públicos se puede y se debe desarrollar a través de las APP.

Respecto a la inversión a un nivel más general, en Perú se ha impulsado la inversión privada a través de adjudicaciones de proyectos bajo la modalidad de asociaciones 
público-privadas (APP), las cuales, desde mediados del año 2011 hasta inicios del año 2017, han sido por un monto superior a los 16000 millones de dólares. (Bonifaz et al., 2020, p. 9)

Y es en las APP en donde el modelo de financiamiento imperante es el project finance o alguna modalidad similar de financiamiento. Es por esto que es importante conocer el project finance para que, sobre esta base, se diseñen las políticas públicas que permitan mantener aquellos aspectos positivos del marco legal e institucional que facilitan la ejecución de proyectos bajo la modalidad de APP, y corregir y mejorar aquellos aspectos que sean necesarios para destrabar la ejecución de las obras de infraestructura.

\section{LA VENTAJA DE LA EJECUCIÓN DE PROYECTOS BAJO LA MODALIDAD DE APP}

Está claro que las APP no son el único mecanismo para combatir la brecha de infraestructura, pero presentan una ventaja significativa frente a la contratación pública tradicional y las obras por impuestos: la sostenibilidad de la infraestructura y del servicio en el tiempo.

Nos explicamos. Cuando se construye un hospital como obra pública, una empresa constructora ejecuta la obra, la entrega y culmina el vínculo contractual. Luego es el Estado, bien sea a través del Ministerio de Salud o de EsSalud, el encargado de brindar el servicio, mantener la infraestructura, el equipamiento y, en general, adquirir los repuestos, los insumos, hacer los análisis de laboratorio, etcétera.

Existen abundantes experiencias que demuestran que cuando los hospitales son administrados por el Estado el servicio es deficiente. Los equipos se deterioran y dejan de funcionar, los insumos se agotan, los reactivos escasean, la limpieza no se realiza como es debido... En suma, el servicio brindado a los usuarios es muy pobre. Esto se vio con mayor claridad aún con motivo de la pandemia del COVID-19. Se detectó que algunos hospitales tenían equipos inoperativos, sin mencionar la falta de una adecuada organización para brindar el servicio.

En cambio, hay experiencias a nivel nacional e internacional que demuestran que un hospital bajo la modalidad de APP garantiza un servicio enormemente superior al de un hospital público. Los equipos funcionan, los laboratorios atienden con normalidad, no faltan reactivos, no faltan medicinas, no se cancelan frecuentemente las citas, se mantiene la limpieza, etcétera. Una APP es, como ya dijimos, un compromiso de largo plazo. En una APP la empresa privada no construye un hospital y se va. En una APP la empresa privada diseña, construye y equipa un hospital, pero además brinda el servicio, da mantenimiento, limpieza, alimentación, e incluso se encarga del servicio médico cuando el contrato lo prevé. En el Perú tenemos como ejemplo los hospitales Alberto Barton del Callao y Guillermo Kaelin de Villa María del Triunfo. 
Obviamente, para lograr y consolidar estos beneficios de las APP se deben diseñar los contratos manteniendo los incentivos y desincentivos adecuados. Debe existir una adecuada supervisión que vigile el cumplimiento de la calidad del servicio por parte de la SPV, siendo idealmente medida a través de indicadores objetivos (por ejemplo, cantidad de pacientes a quienes no se les entregó las medicinas que fueron recetadas por el médico, la cual debería ser de cero o casi cero), así como también debe haber un mecanismo claro de penalidades y deducciones a que se hace acreedora la SPV en caso de incumplimiento. Cabe mencionar también el mantenimiento de una garantía de fiel cumplimiento por un monto significativo, la cual será ejecutada en caso de incumplimiento que derive en una resolución del contrato.

Pero, nuevamente, para que sean viables las APP se debe comenzar, como piedra angular, por estructurar proyectos que sean bancables. De nada serviría estructurar un contrato que esté sobregarantizado y que establezca abundantes deducciones y penalidades si este no es bancable. Si no es susceptible de obtener financiamiento, no se podrá ejecutar la obra y, por consiguiente, no se podrá brindar el servicio.

From the Contracting Authority's perspective, the bankability of a PPP project is often a key consideration in determining if an infrastructure project can be procured using a PPP approach. However, governments should not just consider bankability, but also value for money and robust risk allocation. i.e. a project can be bankable, but not deliver value for money because a Contracting Authority is transferring risks to the private sector that could be more efficiently managed by the government. PPP is not a procurement method which transfers all risk to the Private Partner. There will always be some risks for which the Contracting Authority should be wholly or partly responsible. (Global Infrastructure Hub, 2019, p. 7)

\section{CONCLUSIONES}

Resulta claro que tenemos una gran brecha de infraestructura que necesitamos combatir y que uno de los mecanismos más apropiados para ello son las APP. Asimismo, la experiencia nacional e internacional nos demuestra que el financiamiento de proyectos de APP se hace, principalmente, a través del project finance.

En consecuencia, es importante difundir las bondades de esta forma de financiamiento y realizar las acciones necesarias para que continúen ejecutándose proyectos de infraestructura sustentados en el project finance y bajo la modalidad de APP, pues ello redundará en la mejora de la calidad de vida de la población y en convertirnos en un país más competitivo en un mundo globalizado. 


\section{REFERENCIAS}

Bonifaz, J. L., Urrunaga, R., Aguirre, J., y Quequezana, P. (2020). Brecha de infraestructura en el Perú: estimación de la brecha de largo plazo 2019-2038. Banco Interamericano de Desarrollo. https://publications.iadb.org/publications/spanish/document/ Brecha-de-infraestructura-en-el-Peru-Estimacion-de-la-brecha-de-infraes tructura-de-largo-plazo-2019-2038.pdf

Casanovas, T. (2016). Project Finance Internacional. Profit Editorial.

Global Infrastructure Hub. (2018). Leading Practices in Governmental Processes Facilitating Infrastructure Project Preparation. https://library.pppknowledgelab.org/documents/document-group/108

Global Infrastructure Hub. (2019). PPP Risk Allocation Tool 2019 Edition. Social Infrastructure. https://cdn.gihub.org/umbraco/ media/3018/gih_rat_social_infrastructure_art_web.pdf

Jiménez, M. E. (2015). La sencillez como cualidad moral en las exposiciones científicas orales. MEDISAN, 19(2), 273-287. http://scielo.sld.cu/scielo.php?script=sci_artte $x t \& p i d=S 1029-30192015000200018$

Nalvarte Salvatierra, P. (2018). Evolución del marco legal de las concesiones y las asociaciones público-privadas en el Perú. Derecho \& Sociedad, 49, 359-379.

Reátegui, A., Carranza, I., Galván, J. P., y Gutiérrez, G. (2018). Project finance para proyectos de APP en el Perú: evaluación tributaria, contable y financiera. ESAN Ediciones.

The World Bank (2019). Guidance on PPP Contractual Provisions. https://ppp.worldbank.org/ public-private-partnership/library/guidance-ppp-contractual-provisions-2019 